\title{
USO DE PROBIÓTICOS SISTÊMICOS NO TRATAMENTO PERIODONTAL NÃO CIRÚRGICO: REVISÃO NARRATIVA DA LITERATURA
}

\author{
USE OF SYSTEMIC PROBIOTICS IN NON-SURGICAL PERIODONTAL TREATMENT: \\ NARRATIVE LITERATURE REVIEW
}

Renata Queiroz Tavares ${ }^{1}$, Mariana Nogueira de Figueiredo², Willy Bustillos Torrez ${ }^{3}$, Cristiana Fernandes Plutarco Nogueira ${ }^{4}$, Angelo Ferrari Roberto ${ }^{5}$, Karyne Maria Rossit Silva Kiausinis ${ }^{6}$, Magda Feres ${ }^{7}$, Belén Retamal-Valdes ${ }^{8}$

\begin{abstract}
RESUMO
O objetivo do presente estudo foi avaliar a evidência científica atualmente disponível sobre a utilização de probióticos sistêmicos como adjuvantes à raspagem e alisamento radicular (RAR) no tratamento da periodontite em relação aos parâmetros clínicos, microbiológicos e imunológicos. Para tal, realizou-se uma revisão crítica da literatura em busca de estudos clínicos que utilizassem probióticos como adjuntos à RAR no tratamento da periodontite. Diferentes espécies bacterianas têm sido utilizadas como probióticos, sendo o mais citado o Lactobacillus reuteri. Foram obtidos resultados clínicos positivos na redução do acúmulo de biofilme e no índice gengival, na redução da profundidade de sondagem e no ganho do nível clínico de inserção com a utilização dos probióticos. Os probióticos mostraram ainda efeito favorável na manutenção da microbiota compatível com saúde e na modulação da resposta imunológica. O uso adjunto de probióticos à RAR parece ser mais acentuado em casos de periodontite associada a bolsas moderadas e profundas. Esses efeitos podem diminuir a necessidade da segunda fase cirúrgica do tratamento periodontal. Porém, as evidências científicas ainda são insuficientes para a recomendação do uso de probióticos na prática clínica periodontal. Mais estudos ainda são necessários para se determinar: resultados clínicos no longo prazo, melhores cepas para se obter os melhores resultados, dose, via/veículo de administração e tempo de tratamento ideal.
\end{abstract}

PALAVRAS-CHAVE: Periodontite. Probióticos. Terapia

\begin{abstract}
The aim of this study was to evaluate the currently available scientific evidence on the use of systemic probiotics as adjuvants to scaling and root planning (SRP) in the treatment of periodontitis in terms of clinical, microbiological and immunological parameters. A critical review of the literature was conducted in search of clinical studies that used probiotics as adjuncts to SRP in the treatment of periodontitis. Different bacterial species have been used as probiotics, and Lactobacillus reuteri was the most cited. Positive clinical results were obtained in reducing plaque and gingival indexes and probing depth, and gain in clinical attachment level with the use of probiotics. Probiotics also showed a favorable effect in maintaining a health-compatible microbiota and positively modulated the immune response. The adjunct use of probiotics to SRP seems to be stronger in cases of moderate and deep pockets. These effects may decrease the need for the surgical phase of periodontal treatment. However, scientific evidence is still insufficient to recommend the use of probiotics in periodontal clinical practice. Further studies are still needed to determine: long-term clinical results, best strains to obtain the best results, dose, route/vehicle of administration and ideal treatment time.

\footnotetext{
${ }^{1}$ Cirurgiã dentista, especialista em periodontia, especialista em ortodontia, mestranda em odontologia.

${ }^{2}$ Cirurgiã dentista, mestrado em periodontia, doutoranda em odontologia.

${ }^{3}$ Cirurgião dentista, mestrado em ciências biomédicas, doutorando em odontologia.

4 Cirurgiã dentista, especialista em prótese dental, mestre em implantodontia, doutorando em odontologia.

5 Cirurgião dentista, especialista em periodontia/harmonização orofacial, mestrando em odontologia.

${ }^{6}$ Cirurgiã dentista, especialista em implantodontia, mestranda em odontologia.

7 Cirurgiã dentista, especialista em periodontia, mestrado em periodontia, doutorado em ciências médicas e biologia oral

${ }^{8}$ Cirurgiã dentista, mestrado em odontologia (área de concentração em periodontia), doutorado em odontologia (área de concentração em periodontia).
} 
USO DE PROBIÓTICOS SISTÊMICOS NO TRATAMENTO PERIODONTAL NÃO CIRÚRGICO:
REVISÃO NARRATIVA DA LITERATURA Renata Queiroz Tavares, Mariana Nogueira de Figueiredo, Willy Bustillos Torrez, Cristiana Fernandes Plutarco Nogueira, Angelo Ferrari Roberto, Karyne Maria Rossit Silva Kiausinis, Magda Feres, Belén Retamal-Valdes

KEYWORDS: Periodontitis. Probiotics. Treatment

\section{INTRODUÇÃO}

A periodontite é uma doença infectoinflamatória crônica e multifatorial caracterizada pela interação entre uma microbiota disbiótica e a resposta imune do hospedeiro, e leva à destruição dos tecidos de suporte dentais $(1,2)$. O diagnóstico da periodontite se baseia na identificação de parâmetros clínicos, como aumento na profundidade de sondagem (PS), perda de inserção clínica, perda óssea alveolar e presença de sangramento à sondagem (3). A progressão da periodontite pode ser modulada por fatores de risco como fumo e diabetes (8). Outros indicadores de risco têm sido relatados, tais como aspectos comportamentais (stress), estilo de vida (obesidade e nutrição) e por condições sistêmicas (osteoporose e HIV) $(9,10,11)$. A periodontite também pode constituir um risco à saúde sistêmica do indivíduo $(4,5,6,7)$.

Durante quase 100 anos, dificuldades técnicas em se avaliar a complexa microbiota subgengival, composta por patógenos anaeróbios estritos, atrasaram o diagnóstico e consequentemente 0 tratamento da periodontite. Técnicas de biologia molecular, como PCR (Polymerase Chain Reaction) e sondas de DNA tiveram um papel primordial na identificação e quantificação bacteriana associada com as doenças periodontais (12). Utilizando a técnica checkerboard DNA-DNA hybridization, Socransky e colaboradores descreveram os denominados "Complexos Microbianos" no ambiente subgengival. As espécies compatíveis com saúde (primeiras colonizadoras) foram agrupadas nos complexos amarelo, roxo, verde e no grupo Actinomyces; já as espécies patogênicas foram agrupadas no complexo laranja e vermelho. O complexo vermelho inclui as principais espécies relacionadas à periodontite: Porphyromonas gingivalis, Tannerella forsythia e Treponema denticola (13). Na saúde periodontal, esses complexos coexistem de forma harmônica, com maior proporção de espécies compatíveis com saúde do que as associadas a doenças. (14). Contudo, o acúmulo de biofilme propicia uma mudança ecológica, com aumento na proporção dos complexos patogênicos em relação aos compatíveis com saúde, resultando na disbiose periodontal $(13,15)$. Esse entendimento de que nem todas as espécies bacterianas existentes na cavidade oral são patogênicas é de suma importância para o restabelecimento e manutenção da saúde periodontal (16).

O desequilíbrio na proporção dos complexos microbianos e a interação das espécies entre si e com o hospedeiro estão diretamente relacionados com os parâmetros clínicos de inflamação da periodontite, uma vez que a produção descontrolada de citocinas pró-inflamatórias leva a um aumento da patogenicidade e da destruição tecidual 
$(17,13,18)$. Uma abordagem clínica efetiva é aquela que viabiliza o retorno de proporções bacterianas compatíveis com saúde, interrompe a progressão da doença e cria condições que ajudarão o paciente a manter uma dentição saudável, funcional e confortável a longo prazo (3).

A terapia periodontal não cirúrgica é o tratamento mais usado para o manejo da periodontite (19), consiste principalmente em instrução de higiene oral e raspagem e alisamento radicular (RAR) para o controle do biofilme (20). No entanto, em casos de periodontite avançada ou severa, a RAR sozinha nem sempre é eficaz, possivelmente por não conseguir levar a rebiose do biofilme na cavidade oral (21). Sendo assim, outras terapias têm sido propostas para otimizar os resultados da RAR no manejo da periodontite, como por exemplo, terapia fotodinâmica (22), laser de baixa potência (23) e a administração sistêmica de antimicrobianos (24) ou de probióticos $(26,27)$. A combinação de metronidazol (MTZ) e amoxicilina (AMX) sistêmicos à RAR tem mostrado ser um tratamento periodontal promissor (25). Contudo, os efeitos colaterais e a possível resistência bacteriana associada à prescrição desses medicamentos, têm motivado os pesquisadores na busca por novas alternativas terapêuticas, por exemplo, o uso de probióticos sistêmicos $(26,27)$.

O objetivo desta revisão narrativa da literatura é descrever e discutir a evidência científica disponível sobre a utilização de probióticos como adjuvantes no tratamento periodontal não-cirúrgico na alteração de parâmetros clínicos, microbiológicos e imunológicos.

\section{REVISÃO NARRATIVA DA LITERATURA}

\section{O que sabemos sobre probióticos} 2.10 que são os probióticos?

Segundo a Organização Mundial da Saúde, os probióticos são "microrganismos vivos que, quando administrados em quantidades adequadas, conferem benefícios à saúde do hospedeiro a partir da supressão de patógenos endógenos e exógenos, beneficiando a resposta imune" (28).

A utilização de microrganismos em alimentos vem desde a antiguidade (29). Os probióticos foram propostos pela primeira vez para melhoria na saúde gastrointestinal por Elie Metchnikoff, (Prêmio Nobel de Fisiologia e Medicina - 1908) (26). Desde então, seus efeitos na saúde humana têm sido comprovados em diversos trabalhos que relatam 0 uso de cepas probióticas na manutenção do equilíbrio nos ecossistemas gastrointestinal, geniturinário e oral $(30,31,32)$.

\subsection{Mecanismo de ação dos Probióticos.}

Embora o mecanismo de ação dos probióticos na cavidade oral não esteja precisamente elucidado, ele pode variar de acordo com a cepa ou combinações de cepas utilizadas, a tensão e concentração dos probióticos, o veículo de apresentação, a presença de prebióticos (substratos utilizados 


\section{RECIMA21 - REVISTA CIENTÍFICA MULTIDISCIPLINAR}

USO DE PROBIÓTICOS SISTÊMICOS NO TRATAMENTO PERIODONTAL NÃO CIRÚRGICO: REVISÃO NARRATIVA DA LITERATURA Renata Queiroz Tavares, Mariana Nogueira de Figueiredo, Willy Bustillos Torrez, Cristiana Fernandes Plutarco Nogueira, Angelo Ferrari Roberto, Karyne Maria Rossit Silva Kiausinis, Magda Feres, Belén Retamal-Valdes

por microrganismos hospedeiros que conferem benefícios à saúde), a condição a ser tratada, bem como 0 estágio de progressão da periodontite $(26,34)$.

Os efeitos locais e sistêmicos dos probióticos no tratamento periodontal podem estar relacionados a 3 principais vias $(35,36,27,37)$ :

a. Mecanismo de exclusão competitiva (Ação indireta)

Os probióticos competem com patógenos por nutrientes essenciais e por superfície de adesão, o que resulta em mudança da composição do biofilme e modulação do pH (26).

b. Produção de substâncias antimicrobianas

(Ação direta)
Os probióticos produzem ácido láctico, peróxido de hidrogênio e bacteriocinas, que podem destruir ou inibir o crescimento de patógenos periodontais (38).

\section{c. Ação sobre o hospedeiro}

- Modulação da resposta imune inata e adaptativa: reduzindo a produção de citocinas pró-inflamatórias, tais como IL-6, IL$1 \beta$, fator de necrose tumoral (TNFa), e aumentando a produção de citocinas antiinflamatórias, como a IL-10 (39);

- Indução de expressão de proteínas citoprotetoras nas superfícies das células hospedeiras (26);

- Inibição de colagenases e redução de moléculas associadas à inflamação (26).

\section{A Figura 1 descreve os mecanismos plausíveis pelos quais as espécies probióticas podem} afetar positivamente a saúde periodontal. 


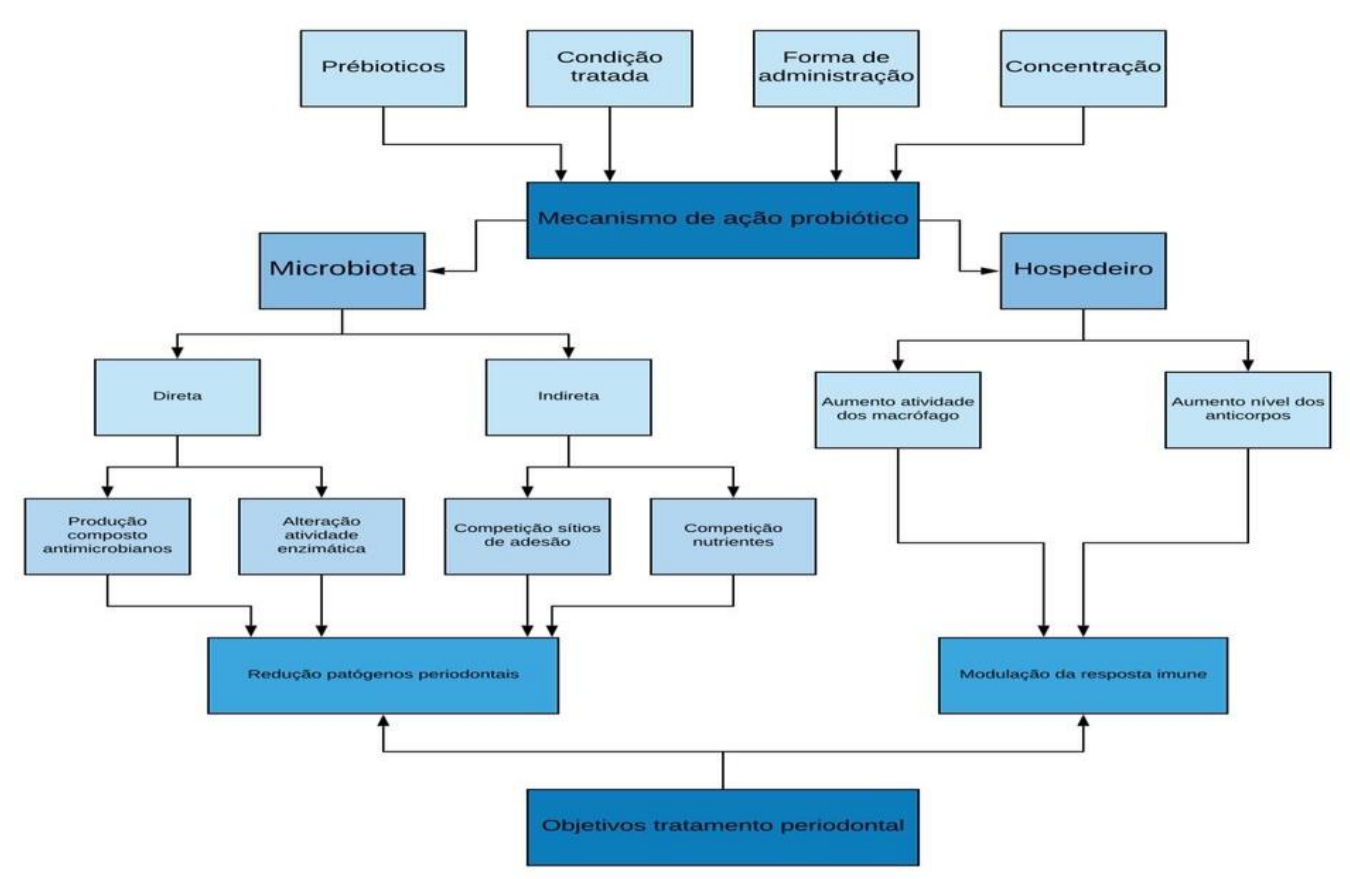

Figura 1. Esquema com os mecanismos de ação plausíveis pelos quais as espécies probióticas podem afetar positivamente a saúde periodontal

\subsection{Principais espécies usadas como probióticos}

Os probióticos são cepas bacterianas geralmente isoladas da microbiota comensal humana. As espécies mais utilizadas de probióticos pertencem aos gêneros Lactobacillus, Bifidobacterium, Escherichia, Enterococcus Streptococcus e Bacillus, sendo todos residentes da pele humana, trato gastrointestinal, trato respiratório e genital feminino (41).

\section{Lactobacillus}

Espécies do gênero Lactobacillus são grampositivas e anaeróbias facultativas. Algumas cepas utilizadas no tratamento adjunto a RAR são: Lactobacillus acidophilus,

Lactobacillus reuteri, Lactobacillus bulgaricus, Lactobacillus rhamnosus, Lactobacillus salivarius, e Lactobacillus casei (42). Dentre essas cepas, a mais estudada é a L. reuteri 
USO DE PROBIÓTICOS SISTÊMICOS NO TRATAMENTO PERIODONTAL NÃO CIRÚRGICO:
REVISÃO NARRATIVA DA LITERATURA Renata Queiroz Tavares, Mariana Nogueira de Figueiredo, Willy Bustillos Torrez, Cristiana Fernandes Plutarco Nogueira, Angelo Ferrari Roberto, Karyne Maria Rossit Silva Kiausinis, Magda Feres, Belén Retamal-Valdes

(43). Embora seu mecanismo de ação na periodontite ainda não tenha sido completamente elucidado, três mecanismos de ação são sugeridos: secreção de bacteriocinas (reuterina e reutericiclina), competição com bactérias patogênicas pela adesão tecidual, e inibição da secreção de citocinas pró-inflamatória $(44,45)$.

\section{Bifidobacterium}

Espécies do gênero Bifidobaterium spp. são gram-positivas e anaeróbicas (46). As espécies mais conhecidas são: Bifidobaterium lactis, Bifidobaterium breve, Bifidobaterium longum, e Bifidobaterium infantis (42).

Estudos in vitro demonstraram que cepas do gênero Bifidobacterium podem aderir fortemente ao biofilme subgengival e reduzir significativamente a contagem de $P$. gingivalis (47). Estudos em animais demonstraram um efeito protetor contra a perda óssea alveolar e a perda de inserção do tecido conjuntivo (48) e a potencialização dos efeitos da RAR pelo B. lactis HN019 na periodontite experimental em ratos (49). Benefícios positivos também foram observados em estudos clínicos, tanto nos parâmetros clínicos, quanto nos microbiológicos e imunológicos (50).

\section{Streptococcus}

Espécies do gênero Streptococcus spp. são gram-positivas e anaeróbias facultativas. São abundantes na cavidade oral e estão relacionadas com a saúde bucal (13). Após a RAR, Streptococcus spp. recolonizam rapidamente a bolsa periodontal (51), podendo retardar a recolonização por bactérias relacionadas com a periodontite (52). Duas cepas de Streptococcus comensais orais, Streptococcus cristatus e Streptococcus salivarius, foram capazes de inibir a secreção IL-8 induzida por bactérias periodontopatogênicas, indicando um efeito benéfico na manutenção da saúde periodontal (53).

\subsection{Formas de Apresentação}

Das diversas formas de administração dos probióticos, a pastilha tem sido mais frequentemente usada nos estudos clínicos que avaliam probióticos como adjunto no tratamento da periodontite $(45,50,54,55,56,57,58,59,60)$ (Tabela 2). Esta apresentação parece ser a mais eficaz por ter efeito local (diluída na cavidade oral) e sistêmico (deglutição com a saliva). Porém, até o momento não há um consenso sobre qual forma de administração é a mais indicada.

\subsection{Uso em Medicina}

Tradicionalmente, os probióticos têm sido usados como um recurso auxiliar a diversos tipos de terapias, sobretudo na gastroenterologia (61). Resultados positivos são relatados com o uso de probióticos na prevenção da diarreia associada a antibióticos (62), síndrome do intestino irritável (63), intolerância a lactose (64), indigestão (65) e 
inchaço estomacal (66).

Os probióticos

também podem exercer outros efeitos favoráveis no tratamento de doenças crônicas, como alguns tipos de câncer, colesterol sérico elevado, quadros alérgicos como rinite e asma, HIV, bem como candidíase vulvovaginal (67).

\subsection{Uso em Odontologia}

Além do efeito potencial no tratamento das doenças periodontais, o uso de probióticos tem sido estudado em outras áreas da odontologia, tais como:

\section{Cárie}

Os efeitos dos probióticos sobre 0 tratamento da cárie têm sido extensivamente investigados em diversos estudos experimentais, utilizando diferentes cepas como: L. rhamnosus GG, L. casei, L. reuteri, $L$. plantarum, Lactobacillus brevis CD2, Bifidobacterium spp e Streptoccus spp. Os principais benefícios avaliados são a redução da presença de Streptococcus mutans e Lactobacillus, controle de $\mathrm{pH}$ da placa e paralisação de lesões de cáries $(68,69)$.

L. rhamnosus $\mathrm{GG}$ é um probiótico que não fermenta sacarose nem lactose e tem demonstrado reduzir significativamente o risco de cárie $(70,71)$. Entretanto, esse probiótico não permanece presente na cavidade oral por mais de 14 dias (72). Sendo assim, outros probióticos continuam sendo avaliados em relação a ação sobre a redução da cariogenicidade do biofilme oral (73).
Até o presente momento, não há evidência científica suficiente de que os probióticos são capazes de prevenir a cárie, embora eles sejam eficazes em reduzir o número de $S$. mutans $(31,73)$.

\section{Candidíase}

A prevalência de candidíase oral tem crescido devido a fatores predisponentes como o uso de próteses removíveis, fumo, doenças crônicas como diabetes, HIV e câncer oral (74). O uso de probióticos desempenha um papel protetor em relação a colonização por Candida spp, principalmente em pacientes que fazem uso de próteses removíveis (75).

\section{Mucosite Oral}

O uso de probióticos pode reduzir a incidência e severidade da mucosite oral, efeito colateral adverso comum de muitas modalidades de terapias oncológicas como quimioterapia, radioterapia e quimioterapia, que pode causar dor, ulceração, disfagia, desnutrição e até interrupção do tratamento. Os resultados são animadores, entretanto, são necessários mais estudos clínicos para sustentar essa indicação (76).

\section{Halitose}

A halitose é um problema crônico com tratamento desafiador. A maior parte dos casos apresenta origem oral, decorrente de compostos voláteis de enxofre (volatile sulfur compounds, VSC) e compostos 
organolépticos voláteis (organoleptic test, OLT) (77). O uso de probióticos tem mostrado resultados positivos (78) moderados no controle da halitose em relação aos escores de OLT, mas não significativos na redução de VSC. Logo, os dados disponíveis ainda são insuficientes para a definição de um protocolo de uso de probióticos no tratamento da halitose (79).

\subsection{Probióticos como adjuntos à RAR no Tratamento Periodontal}

\section{As Tabela 1 e Tabela 2} apresentam as principais características dos estudos clínicos publicados testando diferentes cepas de probióticos como adjuntos à RAR no tratamento da periodontite comparados à RAR sozinha. Os efeitos clínicos, microbiológicos e imunológicos dos probióticos foram evidenciados nessas Tabelas.

\subsubsection{Resultados Clínicos}

\section{Índice de placa}

Embora alguns autores relatem uma diminuição significativa do índice de placa (acúmulo de biofilme) $(45,54,56,57,58,50)$ com o uso de probióticos, outros não observaram esse benefício $(55,80,59,81,60)$.

\section{Índice Gengival (82)}

A maioria dos estudos que avaliaram o índice gengival, mostrou uma melhora deste parâmetro clínico quando o probiótico foi utilizado como terapia adjuvante à RAR $(45,54,56,58)$.

\section{Sangramento à sondagem}

No que diz respeito ao sangramento à sondagem, os resultados são heterogêneos. Enquanto alguns estudos relataram que o uso de Lactobacillus está relacionado a diminuição significativa desse parâmetro clínico $(50,54,55,56,58)$, outros não conseguiram relatar essa associação $(45,57,80,81,60)$.

\section{Profundidade de sondagem}

O uso de probióticos no tratamento periodontal tem levado a reduções significativas na PS, especialmente em bolsas moderadas $(56,58)$ e profundas $(50,54,80)$. Contudo, esse benefício não foi observado quando avaliado em conjunto as diferentes profundidades de bolsa (rasas, moderadas e profundas) $(57,59,81,60)$.

\section{Nível de inserção clínica}

Semelhante ao resultado observado na PS, os ganhos de inserção clínica com o uso de probióticos foram estatisticamente significativos maiores dos que os observados com RAR somente, mas somente em bolsas moderadas e profundas $(50,54,80)$.

\section{Necessidade de cirurgia (83) e Risco de progressão da doença (84)}

O uso de probióticos também pode contribuir para um menor número de bolsas residuais pós-terapia (50), baixo risco de progressão da doença periodontal $(58,50,80)$ e menor necessidade de terapia adicional $(58,50)$. 


\title{
RECIMA21 - REVISTA CIENTÍFICA MULTIDISCIPLINAR
}

\author{
USO DE PROBIÓTICOS SISTÊMICOS NO TRATAMENTO PERIODONTAL NÃO CIRÚRGICO: \\ REVISÃO NARRATIVA DA LITERATURA \\ Renata Queiroz Tavares, Mariana Nogueira de Figueiredo, Willy Bustillos Torrez, Cristiana Fernandes Plutarco Nogueira, \\ Angelo Ferrari Roberto, Karyne Maria Rossit Silva Kiausinis, Magda Feres, Belén Retamal-Valdes
}

Em resumo, o uso adjunto de probióticos à RAR mostra ser eficaz na redução do acúmulo de placa e do índice gengival, parece ser mais indicado em casos de periodontite com bolsas moderadas e profundas e apresentou resultados positivos nas bolsas residuais (PS> 4mm) após a RAR com melhora na PS e no NIC. Esses efeitos podem diminuir a necessidade da fase cirúrgica do tratamento periodontal $(43,85,86)$.

\subsubsection{Resultados microbiológicos}

As técnicas microbiológicas mais frequentemente utilizadas nos estudos testando probióticos no tratamento periodontal tem sido: $\quad$ cultura $(45,57,58,59), \quad$ PCR $(54,81)$ e checkeboard DNA-DNA hybridization (50). As principais evidências a partir dos resultados desses estudos estão resumidas a seguir:

\section{Lactobacillus}

O L. reuteri demostrou redução eficaz de potentes patógenos periodontais, como $P$. gingivalis $(45,54)$, Prevotella intermedia e Aggregatibacter actinomycetemcomitans (A. Actinomycetemcomitans) presentes no biofilme subgengival (45). Quando avaliada a ação deste probiótico no biofilme supra gengival e na saliva houve uma redução de $P$. gingivalis e $P$. intermedia. Entretanto, não houve diferença nos níveis de $A$. actinomycetemcomitans, Fusobacterium nucleatum, e T. Forsythia (54). Outro benefício deste probiótico é a sua permanência na bolsa periodontal por até 180 dias após o início do tratamento (58).

O uso isolado de L. rhamnosus parece não ser suficiente para reduzir de forma significativa de $P$. gingivalis, A.actinomycetemcomitans e $T$. forsythia na placa subgengival (81).

\section{Bifidobacterium}

Bifidobacterium animalis ss lactis ( $B$. lactis) está relacionado a redução de bactérias periodontopatogênicas, tanto do complexo vermelho quanto do complexo laranja por $30 \mathrm{e}$ 90 dias após o tratamento, respectivamente. Além disso, essas cepas favorecem o aumento de Actinomyces spp (complexo azul) e Streptococcus spp, microrganismos compatíveis com saúde periodontal $(50,48)$. A composição subgengival dos complexos vermelho e laranja parecem retornar às proporções iniciais em 360 dias (50).

\section{Streptococcus}

A combinação de $S$. oralis $\mathrm{KJ} 3, S$. uberis $\mathrm{KJ} 2$ e $S$. rattus $\mathrm{JH} 145$ pode promover redução significativa de $P$. gingivalis na saliva (57).

\section{Associações}

A fim de amplificar os benefícios periodontais, associações de diferentes cepas probióticas têm sido propostas com o intuito de somar seus resultados positivos. A associação de $L$. sporogenes, 
Streptococcus faecalis, Clostridium butyricum

e Bacillus mesentericus mostrou redução estatística no número de $P$. gingivalis no biofilme subgengival, mas não para $A$. actinomycetemcomitans e $P$. intermedia (59).

Quando L. rhamnosus ao B. lactis

BB12 foram associados, houve redução significativa de $P$. gingivalis, $F$. nucleatum $e$ Aggregatibacter actinomycetemcomitans no biofilme supragengival; além dos dois últimos também na saliva. Entretanto, não houve diferença estatística para $P$. intermedia (87).

\subsubsection{Resultados Imunológicos}

Somente três estudos clínicos avaliaram também os efeitos imunomoduladores dos probióticos $(50,55,56)$. Todos os estudos utilizaram o método ELISA (ensaio de imunoabsorção enzimática) e análise do fluido crevicular gengival (FCG) $(50,56,55)$.

O L. reuteri levou a uma redução na produção de citocinas pró-inflamatórias (TNF$\alpha, I L-1 \beta, I L-17)$ (55). No acompanhamento de 1 ano, essa espécie proporcionou um nível significativamente menor de metaloproteinase8 da matriz (MMP-8) e do volume de FCG, por até 180 dias. Além disso, foi observado um aumento no nível do inibidor de tecido da metaloproteinase 1 da matriz (TIMP-1) e de citocinas anti-inflamatórias pelo mesmo período (56).

Outra espécie avaliada foi 0 Bifidobacterium, que levou a um maior nível de interleucinas IL-10 em 30 dias, menor de IL-1 $\beta$ em 30 e 90 dias, e menor de IL-8 em 30 dias (50).

\section{DISCUSSÃO}

Essa revisão narrativa da literatura buscou avaliar a evidência científica atual sobre o uso de probióticos sistêmicos como adjuntos à RAR no tratamento da periodontite, baseada nos estudos clínicos aleatorizados disponíveis até o momento. Clinicamente, o uso adjunto de probióticos promove maior diminuição da profundidade de sondagem em bolsas periodontais inicialmente moderadas $\mathrm{e}$ profundas, quando comparado à RAR sozinha. Além disso, a capacidade de reduzir - acúmulo de biofilme supragengival e o índice gengival pode aumentar a manutenção da saúde periodontal por um período prolongado. Microbiologicamente, os grupos que usaram probióticos mostraram ainda efeito favorável na manutenção da microbiota compatível com saúde, que perdurou por mais tempo do que a RAR isoladamente. Finalmente, o uso de probióticos parece modular positivamente a resposta imunológica do indivíduo, podendo minimizar a destruição tecidual.

Resultados clínicos com diferentes graus de sucesso foram observados nos estudos clínicos. Porém, de forma geral, todos os estudos mostraram algum benefício quando 0 probiótico foi utilizado $(58,50,56,80,45,54)$. Espécies diferentes de probióticos podem levar às diferenças de 
benefícios observadas nos diversos estudos.

L. reuteri, por exemplo, levou a resultados positivos até 1 ano pós-tratamento $(58,56), 0$ que já não aconteceu com a espécie $L$. rhamnosus (80). Os estudos sugerem ainda que indivíduos com maior probabilidade de doença severa podem ter benefício adicional com o uso adjunto dos probióticos à RAR, uma vez que os dados revelam melhora significativamente maior em bolsas moderadas e profundas $(58,50,56,80,85)$. Esse efeito pode diminuir a necessidade da realização de cirurgias anti-infecciosas durante 0 tratamento periodontal $(58,50,80)$. Além disso, o uso de probiótico na reinstrumentação para tratamento de bolsas residuais, parece potencializar a melhora clínica desses sítios remanescentes $(43,85,86)$.

Apesar de não diminuir a quantidade de bactérias presentes no biofilme, os probióticos podem ser capazes de alterar as proporções bacterianas, ocupando um espaço no biofilme que de outra forma seria ocupado por microrganismos patogênicos (48). Os probióticos foram eficazes em reduzir 0 número de $P$. gingivalis, $T$. denticola, $T$. forsythia e A. actinomycetemcomitans no biofilme $\quad(45,91,87,59,92,50)$. Um maior percentual de patógenos do complexo vermelho foi observado em pacientes não tratados com probióticos, sugerindo que eles podem agir atrasando a maturação do biofilme periodontal associado à doença (50).
Os probióticos parecem desempenhar também 0 papel de moduladores imunológicos, reduzindo a produção de citocinas pró-inflamatórias (TNFa, IL-1 $\beta$, IL-17) (55) e da expressão de MMP-8, a principal colagenase responsável pela destruição tecidual na periodontite (56). Além disso, estudos sugerem um maior nível de citocinas anti-inflamatórias e TIMP-1 (fator modulador da atividade de metaloproteinases) com o uso de probióticos (56).

Os achados deste artigo estão de acordo com revisões sistemáticas recentes que enfatizam que 0 uso de probióticos apresenta resultados positivos nos aspectos clínicos e microbiológicos, a curto prazo, e que o pequeno número de estudos com análises imunológicas impossibilita uma avaliação quantitativa precisa desses efeitos $(93,94)$.

Para implementar uma nova terapia na prática clínica, são necessários estudos clínicos randomizados, com uma amostra robusta (pelo menos 100 participantes) e acompanhamento a longo prazo (pelo menos 1 ano), além de revisões sistemáticas (98). Os estudos clínicos disponíveis aliando os efeitos de probióticos como adjuntos à terapia periodontal apresentaram amostras pequenas, (28 a 48 indivíduos), e com curto tempo de acompanhamento (28 e 360 dias). Assim, embora estudos prévios (in vitro e in vivo) evidenciem plausibilidade biológica para o uso adjunto dos probióticos sistêmicos a RAR no tratamento da periodontite, com um possível efeito modulador da resposta imune 
USO DE PROBIÓTICOS SISTÊMICOS NO TRATAMENTO PERIODONTAL NÃO CIRÚRGICO: REVISÃO NARRATIVA DA LITERATURA Renata Queiroz Tavares, Mariana Nogueira de Figueiredo, Willy Bustillos Torrez, Cristiana Fernandes Plutarco Nogueira, Angelo Ferrari Roberto, Karyne Maria Rossit Silva Kiausinis, Magda Feres, Belén Retamal-Valdes

$(48,49,95)$, ainda existe a necessidade de um maior número de estudos clínicos randomizados com padronização de severidade da doença, cepas, veículo, forma de administração e tempo de utilização para que seja possível se determinar com segurança o benefício adicional do uso de probióticos na prática clínica $(96,93,97)$.

\section{CONCLUSÃO}

O uso adjunto de probióticos sistémicos à RAR tem mostrado efeitos clínicos, microbiológicos e imunológicos favoráveis adicionais aos obtidos com a RAR sozinha. Contudo, as evidências ainda são insuficientes para a sua recomendação clínica.

\section{REFERÊNCIAS}

1. Yucel-Lindberg $T$, Båge $T$. Inflammatory mediators in the pathogenesis of periodontitis. Expert Rev Mol Med. 2013;15.

2. Papapanou PN, Sanz M, Buduneli N, Dietrich $\mathrm{T}$, Feres $\mathrm{M}$, Fine $\mathrm{DH}$, et al. Periodontitis: Consensus report of workgroup 2 of the 2017 World Workshop on the Classification of Periodontal and Peri-Implant Diseases and Conditions. J Periodontol. 2018 Jun 1;89:S173-82.

3. Kinane DF, Stathopoulou PG, Papapanou PN. Periodontal diseases. Nature Reviews Disease Primers. 2017 Jun 22;3:17038.

4. Pihlstrom BL, Michalowicz BS, Johnson NW. Periodontal diseases. In: Lancet. 2005 Nov 19;366(9499):1809-20.

5. Preshaw PM, Alba AL, Herrera D, Jepsen S, Konstantinidis A, Makrilakis
$\mathrm{K}$, et al. Periodontitis and diabetes: $\mathrm{A}$ two-way relationship. Diabetologia. 2012;55:21-31.

6. Lalla E, Papapanou PN. Diabetes mellitus and periodontitis: A tale of two common interrelated diseases. Nature Reviews Endocrinology. 2011;7:73848.

7. Piconi S, Trabattoni D, Luraghi C, Perilli E, Borelli M, Pacei M, et al. Treatment of periodontal disease results in improvements in endothelial dysfunction and reduction of the carotid intima-media thickness. FASEB J. 2009 Apr;23(4):1196-204.

8. Tonetti MS, Greenwell H, Kornman KS. Staging and grading of periodontitis: Framework and proposal of a new classification and case definition. $J$ Periodontol. 2018 Jan;89:S159-72.

9. Gharbi A, Hamila A, Bouguezzi A, Dandana A, Ferchichi S, Chandad F, et al. Biochemical parameters and oxidative stress markers in Tunisian patients with periodontal disease. BMC Oral Health. 2019 Oct 22;19(1).

10. Basher SS, Saub R, Vaithilingam RD, Safii SH, Daher AM, Al-Bayaty FH, et al. Impact of non-surgical periodontal therapy on OHRQoL in an obese population, a randomised control trial. Health Qual Life Outcomes. 2017 Nov;15(1):225.

11. Chapple ILC, Bouchard P, Cagetti MG, Campus G, Carra MC, Cocco F, et al. Interaction of lifestyle, behaviour or systemic diseases with dental caries and periodontal diseases: consensus report of group 2 of the joint EFP/ORCA workshop on the boundaries between caries and periodontal diseases. J Clin Periodontol. 2017;44:S39-51.

12. Socransky SS, Smith C, Martin L, Paster BJ, Dewhirst FE, Levin AE. "Checkerboard" DNA-DNA hybridization. Biotechniques. 1994 Oct;17(4):788-92. 
13. Socransky SS, Haffajee AD, Cugini MA, Smith C, Kent RL. Microbial complexes in subgingival plaque. J Clin Periodontol. 1998;25(2):134-44.

14. Kilian M, Chapple ILC, Hannig M, Marsh PD, Meuric V, Pedersen AML, et al. The oral microbiome - An update for oral healthcare professionals. Br Dent J. 2016 Nov;221(10):657-66.

15. Van Dyke TE, Sima C. Understanding resolution of inflammation in periodontal diseases: Is chronic inflammatory periodontitis a failure to resolve? Periodontology 2000. 2020;82:205-13.

16. Socransky SS, Haffajee AD. Dental biofilms: difficult therapeutic targets. Periodontology 2000. 2002;28:12-55.

17. Bartold PM, Van Dyke TE. Host modulation: controlling the inflammation to control the infection. Periodontology 2000. 2017 Oct;75(1):317-29.

18. Handfield M, Progulske-Fox A, Hillman JD, M JD, Ellen RP, Galimanas VB, et al. Periodontal microbial ecology In vivo induced genes in human diseases Porphyromonas gingivalis, Treponema denticola, and Tannerella forsythia: the \& apos; red complex \& apos; a prototype polybacterial pathogenic consortium in periodontitis Oral microbiology and. Periodontology 2000. 2005;38(1):135-87.

19. Sanz I, Alonso B, Carasol M, Herrera D, Sanz M. Nonsurgical treatment of periodontitis. J Evid Based Dent Pract. 2012 Sep;12(3 Suppl.):76-86.

20. Claffey N, Polyzois I, Ziaka P. An overview of nonsurgical and surgical therapy. Periodontology 2000. 2004;36:35-44.

21. Tomasi $\mathrm{C}$, Leyland $\mathrm{AH}$, Wennström JL. Factors influencing the outcome of nonsurgical periodontal treatment: a multilevel approach. J Clin Periodontol. 2007;34(8):682-90.

22. AlAhmari $\mathrm{F}$, Ahmed $\mathrm{HB}, \mathrm{Al}-$ Kheraif $\mathrm{AA}$,
Javed F, Akram Z. Effectiveness of scaling and root planning with and without adjunct antimicrobial photodynamic therapy in the treatment of chronic periodontitis among cigarette-smokers and never-smokers: A randomized controlled clinical trial. Photodiagnosis Photodyn Ther. 2019 Mar;25:247-52.

23. Zengin Celik T, Saglam E, Ercan C, Akbas F, Nazaroglu K, Tunali M. Clinical and Microbiological Effects of the Use of Erbium: Yttrium-AluminumGarnet Laser on Chronic Periodontitis in Addition to Nonsurgical Periodontal Treatment: A Randomized Clinical Trial-6 Months Follow-Up. Photobiomodulation, photomedicine, laser Surg. 2019 Mar;37(3):182-90.

24. Feres M, Soares GMS, Mendes JAV, Silva MP, Faveri M, Teles $\mathrm{R}$, et al. Metronidazole alone or with amoxicillin as adjuncts to non-surgical treatment of chronic periodontitis: A 1-year doubleblinded, placebo-controlled, randomized clinical trial. $\mathrm{J}$ Clin Periodontol. 2012 Dec;39(12):1149-58.

25. Teughels W, Feres M, Oud V, Martín C, Matesanz P, Herrera D. Adjunctive effect of systemic antimicrobials in periodontitis therapy. A systematic review and meta-analysis. J Clin Periodontol. 2020 Jul 47;(Suppl 22):257-281.

26. Seminario-Amez M, López-López J, Estrugo-Devesa A, Ayuso-Montero R, Jané-Salas $E$. Probiotics and oral health: A systematic review. Medicina Oral, Patologia Oral y Cirugia Bucal. Medicina Oral, Patologia Oral y Cirugia Bucal; 2017;(22):e282-8.

27. Mahasneh S, Mahasneh A. Probiotics: A Promising Role in Dental Health. Dent J. 2017 Sep 27;5(4):26.

28. Meurman JH, Stamatova I. Probiotics: Contributions to oral health. Oral Dis. 2007;13(5):443-51.

29. Salminen MK, Tynkkynen S, Rautelin 
H, Saxelin M, Vaara M, Ruutu P, et al. Lactobacillus Bacteremia during a Rapid Increase in Probiotic Use of Lactobacillus rhamnosus GG in Finland. Clin Infect Dis. 2002 Nov;35(10):1155-60.

30. Laleman I, Teughels W. Probiotics in the dental practice: a review. Quintessence Int [Internet]. 2015 Mar [cited 2020 May 10];46(3):255-64. Available from: http://www.ncbi.nlm.nih.gov/pubmed/25 485319

31. Laleman I, Detailleur V, Slot DE, Slomka V, Quirynen M, Teughels W. Probiotics reduce mutans streptococci counts in humans: A systematic review and meta-analysis. Clinical Oral Investigations. 2014;18:1539-52.

32. Takahashi N. Oral microbiome metabolism: From "who are they?" to "what are they doing?" Journal of Dental Research. 2015;94:1628-37.

33. Alok A, Singh I, Singh S, Kishore $M$, Jha P, lqubal MA. Probiotics: A New Era of Biotherapy. Adv Biomed Res. 2017;6(1):31.

34. Bustamante M, Oomah BD, Mosi-Roa Y, Rubilar M, Burgos-Díaz C. Probiotics as an Adjunct Therapy for the Treatment of Halitosis, Dental Caries and Periodontitis. Probiotics Antimicrob Proteins. 2020 Jun;12(2):325-34.

35. Gueimonde M, Salminen S. New methods for selecting and evaluating probiotics. Dig liver Dis Off J Ital Soc Gastroenterol Ital Assoc Study Liver. 2006 Dec;38(Suppl 2):S242-7.

36. Doron S, Gorbach SL. Probiotics: their role in the treatment and prevention of disease. Expert Rev Anti Infect Ther. 2006 Apr;4(2):261-75.

37. Devine DA, Marsh PD. Prospects for the development of probiotics and prebiotics for oral applications. Journal of Oral Microbiology. 2009;1.

38. Chatterjee A, Bhattacharya H, Kandwal
A. Probiotics in periodontal health and disease. Journal of Indian Society of Periodontology. 2011;15:23-8.

39. Ciorba MA, Stenson WF. Probiotic therapy in radiation-induced intestinal injury and repair. Ann N Y Acad Sci. 2009;1165:190-4.

40. Stamatova I, Meurman JH. Probiotics and periodontal disease. Periodontol 2000. 2009;51:141-51.

41. Floch $\mathrm{MH}$. Recommendations for probiotic use in humans - a 2014 update. Pharmaceuticals. 2014;7(10):999-1007.

42. Turroni F, Ventura M, Buttó LF, Duranti S, O'Toole PW, Motherway MOC, et al. Molecular dialogue between the human gut microbiota and the host: A Lactobacillus and Bifidobacterium perspective. Cellular and Molecular Life Sciences. 2014;71:183-203.

43. Grusovin MG, Bossini S, Calza S, Cappa V, Garzetti G, Scotti E, et al. Clinical efficacy of Lactobacillus reutericontaining lozenges in the supportive therapy of generalized periodontitis stage III and IV, grade C: 1-year results of a double-blind randomized placebocontrolled pilot study. Clin Oral Investig. 2020 Jun;24(6):2015-24.

44. Mu Q, Tavella VJ, Luo XM. Role of Lactobacillus reuteri in human health and diseases. Vol. 9, Frontiers in Microbiology. Frontiers Media S.A.; 2018.

45. Vivekananda MR, Vandana $\mathrm{KL}$, Bhat KG. Effect of the probiotic Lactobacilli reuteri (prodentis) in the management of periodontal disease: A preliminary randomized clinical trial. J Oral Microbiol. 2010;2:1-10.

46. Hojo K, Mizoguchi C, Taketomo N, Ohshima T, Gomi K, Arai T, et al. Distribution of salivary Lactobacillus and Bifidobacterium species in periodontal health and disease. Biosci Biotechnol Biochem. 2007 
Jan;71(1):152-7.

47. Jäsberg $H$, Söderling $E$, Endo $A$, Beighton D, Haukioja A. Bifidobacteria inhibit the growth of Porphyromonas gingivalis but not of Streptococcus mutans in an in vitro biofilm model. Eur J Oral Sci. 2016 Jun;124(3):251-8.

48. Oliveira LFF, Salvador SL, Silva PHF, Furlaneto FAC, Figueiredo L, Casarin $\mathrm{R}$, et al. Benefits of Bifidobacterium animalis subsp. lactis Probiotic in Experimental Periodontitis. J Periodontol. 2017 Feb;88(2):197-208.

49. Ricoldi MST, Furlaneto FAC, Oliveira LFF, Teixeira GC, Pischiotini JP, Moreira ALG, et al. Effects of the probiotic Bifidobacterium animalis subsp. lactis on the non-surgical treatment of periodontitis. A histomorphometric, microtomographic and immunohistochemical study in rats. PLoS One. 2017;12(6):e0179946.

50. Invernici MM, Salvador SL, Silva PHF, Soares MSM, Casarin R, Palioto DB, et al. Effects of Bifidobacterium probiotic on the treatment of chronic periodontitis: A randomized clinical trial. J Clin Periodontol. 2018;45(10):1198210.

51. Jünemann $S$, Prior K, Szczepanowski R, Harks I, Ehmke B, Goesmann A, et al. Bacterial community shift in treated periodontitis patients revealed by ion torrent 16S rRNA gene amplicon sequencing. PLOS One. 2012;7(8):e41606.

52. Teughels W, Newman MG, Coucke W, Haffajee AD, Van Der Mei HC, Kinder Haake S, et al. Guiding periodontal pocket recolonization: A proof of concept. J Dent Res. 2007 Nov;86(11):1078-82.

53. Zhang G, Rudney JD. Streptococcus cristatus attenuates Fusobacterium nucleatum-induced cytokine expression by influencing pathways converging on nuclear factor-kB. Mol Oral Microbiol. 2011 Apr;26(2):150-63.
54. Teughels W, Durukan A, Ozcelik O, Pauwels M, Quirynen M, Haytac MC. Clinical and microbiological effects of Lactobacillus reuteri probiotics in the treatment of chronic periodontitis: A randomized placebo-controlled study. J Clin Periodontol. 2013;40(11):1025-35.

55. Szkaradkiewicz AK, Stopa J, Karpiński TM. Effect of oral administration involving a probiotic strain of Lactobacillus reuteri on proinflammatory cytokine response in patients with chronic periodontitis. Arch Immunol Ther Exp (Warsz). 2014 Dec;62(6):495-500.

56. İnce G, Gürsoy H, İpçi ŞD, Cakar G, Emekli-Alturfan E, Yılmaz S. Clinical and Biochemical Evaluation of Lozenges Containing Lactobacillus reuteri as an Adjunct to Non-Surgical Periodontal Therapy in Chronic Periodontitis. J Periodontol. 2015;86(6):746-54.

57. Laleman I, Yilmaz E, Ozcelik O, Haytac C, Pauwels M, Herrero ER, et al. The effect of a streptococci containing probiotic in periodontal therapy: A randomized controlled trial. $\mathrm{J}$ Clin Periodontol. 2015;42(11):1032-41.

58. Tekce M, Ince G, Gursoy H, Dirikan Ipci S, Cakar G, Kadir T, et al. Clinical and microbiological effects of probiotic lozenges in the treatment of chronic periodontitis: A 1-year follow-up study. J Clin Periodontol. 2015;42(4):363-72.

59. Dhaliwal PK, Grover V, Malhotra R, Kapoor A. Clinical and Microbiological Investigation of the Effects of Probiotics Combined with Scaling and Root Planing in the Management of Chronic Periodontitis: A Randomized, Controlled Study. J Int Acad Periodontol [Internet]. 2017;19(3):1018. Available from: http://www.ncbi.nlm.nih.gov/pubmed/31 473697

60. Pelekos G, Ho SN, Acharya A, Leung WK, McGrath C. A double-blind, paralleled-arm, placebo-controlled and 
randomized clinical trial of the effectiveness of probiotics as an adjunct in periodontal care. J Clin Periodontol. 2019;46(12):1217-27.

61. Ciorba MA. A Gastroenterologist's Guide to Probiotics. Clinical Gastroenterology and Hepatology. 2012;10:960-8.

62. Guo Q, Goldenberg JZ, Humphrey C, El Dib R, Johnston BC. Probiotics for the prevention of pediatric antibioticassociated diarrhea. Cochrane Database Syst Rev. 2019;4:(4).

63. Ford AC, Harris LA, Lacy BE, Quigley EMM, Moayyedi P. Systematic review with meta-analysis: the efficacy of prebiotics, probiotics, synbiotics and antibiotics in irritable bowel syndrome. Aliment Pharmacol Ther. 2018;48(10):1044-60.

64. Oak SJ, Jha R. The effects of probiotics in lactose intolerance: A systematic review. Critical Reviews in Food Science and Nutrition. Taylor and Francis Inc.; 2019;59:1675-83.

65. Verna EC, Lucak S. Use of probiotics in gastrointestinal disorders: What to recommend? Therapeutic Advances in Gastroenterology. SAGE Publications; 2010;3:307-19.

66. Di Stefano M, Miceli E, Armellini E, Missanelli A, Corazza GR. Probiotics and functional abdominal bloating. Journal of clinical gastroenterology. J Clin Gastroenterol; 2004;38.

67. Nazir Y, Hussain SA, Abdul Hamid A, Song Y. Probiotics and Their Potential Preventive and Therapeutic Role for Cancer, High Serum Cholesterol, and Allergic and HIV Diseases. Biomed Res Int. 2018 Aug 29;(2018):3428437.

68. Cagetti MG, Mastroberardino S, Milia E, Cocco F, Lingström P, Campus G. The use of probiotic strains in caries prevention: a systematic review. Nutrients. 2013 Jul;5(7):2530-50.

69. Poorni S, Srinivasan MR, Nivedhitha
MS. Probiotic Streptococcus strains in caries prevention: A systematic review. J Conserv Dent. 2019;22(2):123-8.

70. Meurman JH, Antila $\mathrm{H}$, Korhonen A, Salminen S. Effect of Lactobacillus rhamnosus strain GG (ATCC 53103) on the growth of Streptococcus sobrinus in vitro. Eur J Oral Sci. 1995 Aug;103(4):253-8.

71. Näse L, Hatakka K, Savilahti E, Saxelin M, Pönkä A, Poussa T, et al. Effect of long-term consumption of a probiotic bacterium, Lactobacillus rhamnosus $\mathrm{GG}$, in milk on dental caries and caries risk in children. Caries Res. $2001 ; 35(6): 412-20$.

72. Yli-Knuuttila $\mathrm{H}$, Snäll J, Kari $\mathrm{K}$, Meurman JH. Colonization of Lactobacillus rhamnosus GG in the oral cavity. Oral Microbiol Immunol. 2006 Apr;21(2):129-31.

73. Schwendicke F, Korte F, Dörfer CE, Kneist S, Fawzy El-Sayed K, Paris S. Inhibition of Streptococcus mutans Growth and Biofilm Formation by Probiotics in vitro. Caries Res. 2017;51(2):87-95.

74. Reinhardt LC, Nascente PS, Ribeiro JS, Etges A, Lund RG. A single-center 18-year experience with oral candidiasis in Brazil: a retrospective study of 1,534 cases. Braz Oral Res. 2018;32:e92.

75. Mundula T, Ricci F, Barbetta B, Baccini M, Amedei A. Effect of Probiotics on Oral Candidiasis: A Systematic Review and Meta-Analysis. Nutrients. 2019 Oct;11(10).

76. Jiang $\mathrm{C}$, Wang $\mathrm{H}$, Xia $\mathrm{C}$, Dong $\mathrm{Q}$, Chen $E$, Qiu $Y$, et al. A randomized, doubleblind, placebo-controlled trial of probiotics to reduce the severity of oral mucositis induced by chemoradiotherapy for patients with nasopharyngeal carcinoma. Cancer. 2019 Apr;125(7):1081-90.

77. De Geest S, Laleman I, Teughels W, 
Dekeyser C, Quirynen M. Periodontal diseases as a source of halitosis: a review of the evidence and treatment approaches for dentists and dental hygienists. Periodontol 2000. 2016 Jun;71(1):213-27.

78. Soares LG, Carvalho EB, Tinoco EMB. Clinical effect of Lactobacillus on the treatment of severe periodontitis and halitosis: A double-blinded, placebocontrolled, randomized clinical trial. Am J Dent. 2019 Feb;32(1):9-13.

79. Yoo JI, Shin IS, Jeon JG, Yang YM, Kim JG, Lee DW. The Effect of Probiotics on Halitosis: a Systematic Review and Meta-analysis. Probiotics Antimicrob Proteins. 2019 Mar;11(1):150-7.

80. Morales A, Carvajal $P$, Silva $N$, Hernandez M, Godoy C, Rodriguez G, et al. Clinical Effects of Lactobacillus rhamnosus in Non-Surgical Treatment of Chronic Periodontitis: A Randomized Placebo-Controlled Trial With 1-Year Follow-Up. J Periodontol. 2016;87(8):944-52.

81. Morales A, Gandolfo A, Bravo J, Carvajal P, Silva N, Godoy C, et al. Microbiological and clinical effects of probiotics and antibiotics on nonsurgical treatment of chronic periodontitis: A randomized placebocontrolled trial with 9-month follow-up. J Appl Oral Sci. 2018;26:1-9.

82. Loe H, Silness J. Periodontal disease in pregnancy. Prevalence and severity. Acta Odontol Scand. 1963 Dec;21:53351.

83. Cionca N, Giannopoulou C, Ugolotti G, Mombelli A. Amoxicillin and metronidazole as an adjunct to fullmouth scaling and root planing of chronic periodontitis. J Periodontol. 2009 Mar;80(3):364-71.

84. Lang NP, Tonetti MS. Periodontal risk assessment (PRA) for patients in supportive periodontal therapy (SPT). Oral Health Prev Dent. 2003;1(1):7-16.
85. Iwasaki K, Maeda K, Hidaka K, Nemoto K, Hirose Y, Deguchi S. Daily intake of heat-killed Lactobacillus plantarum L137 decreases the probing depth in patients undergoing supportive periodontal therapy. Oral Heal Prev Dent. 2016;14(3):207-14.

86. Laleman I, Pauwels $M$, Quirynen $M$, Teughels W. A dual-strain Lactobacilli reuteri probiotic improves the treatment of residual pockets: $A$ randomized controlled clinical trial. J Clin Periodontol. 2020 Jan;47(1):43-53.

87. Alanzi A, Honkala S, Honkala E, Varghese A, Tolvanen M, Söderling E. Effect of Lactobacillus rhamnosus and Bifidobacterium lactis on gingival health, dental plaque, and periodontopathogens in adolescents: a randomised placebo-controlled clinical trial. Benef Microbes. 2018 Jun;9(4):593-602.

88. Bartold PM, Van Dyke TE. An appraisal of the role of specific bacteria in the initial pathogenesis of periodontitis. J Clin Periodontol. 2019;46(1):6-11.

89. Marsh PD. Microbial ecology of dental plaque and its significance in health and disease. Advances in dental research. Adv Dent Res. 1994;8:26371.

90. Hasturk $H$, Kantarci A, GoguetSurmenian E, Blackwood A, Andry C, Serhan CN, et al. Resolvin E1 Regulates Inflammation at the Cellular and Tissue Level and Restores Tissue Homeostasis In Vivo. J Immunol. 2007 Nov;179(10):7021-9.

91. Mayanagi G, Kimura M, Nakaya S, Hirata $\mathrm{H}$, Sakamoto $\mathrm{M}$, Benno $\mathrm{Y}$, et al. Probiotic effects of orally administered Lactobacillus salivarius WB21containing tablets on periodontopathic bacteria: a double-blinded, placebocontrolled, randomized clinical trial. J Clin Periodontol. 2009 Jun;36(6):50613.

92. Imran FS, Padmanabhan S, Rao R, 
Suresh A, Bharath D. Evaluation of the efficacy of a probiotic drink containing Lactobacillus casei on the levels of periodontopathic bacteria in periodontitis: A clinico-microbiologic study. Indian J Dent Res Off Publ Indian Soc Dent Res. 2015;26(5):4628.

93. Ho SN, Acharya A, Sidharthan S, Li KY, Leung WK, McGrath C, et al. A Systematic Review and Meta-analysis of Clinical, Immunological, and Microbiological Shift in Periodontitis After Nonsurgical Periodontal Therapy With Adjunctive Use of Probiotics. J Evid Based Dent Pract. 2020;20(1):101397.

94. Vives-Soler A, Chimenos-Küstner E. Effect of probiotics as a complement to non-surgical periodontal therapy in chronic periodontitis: a systematic review. Med Oral Patol Oral Cir Bucal. 2020 Mar;25(2):e161-7.

95. Albuquerque-Souza E, Balzarini D, Ando-Suguimoto ES, Ishikawa $\mathrm{KH}$, Simionato MRL, Holzhausen M, et al. Probiotics alter the immune response of gingival epithelial cells challenged by Porphyromonas gingivalis. J Periodontal Res. 2019 Apr;54(2):11527.
96. Martin-Cabezas R, Davideau JL, Tenenbaum $\mathrm{H}$, Huck O. Clinical efficacy of probiotics as an adjunctive therapy to non-surgical periodontal treatment of chronic periodontitis: A systematic review and meta-analysis. J Clin Periodontol. 2016;43(6):520-30.

97. Ikram S, Hassan N, Raffat MA, Mirza S, Akram Z. Systematic review and metaanalysis of double-blind, placebocontrolled, randomized clinical trials using probiotics in chronic periodontitis. $J$ Investig Clin Dent. 2018 Aug;9(3):e12338.

98. Hadorn DC, Baker D, Hodges JS, Hicks $\mathrm{N}$. Rating the quality of evidence for clinical practice guidelines. J Clin Epidemiol. 1996 Jul;49(7):749-54.

99. Grossi SG, Zambon JJ, Ho AW, Koch G, Dunford RG, Machtei EE, et al. Assessment of risk for periodontal disease. I. Risk indicators for attachment loss. J Periodontol. 1994 Mar; 65(3):260-7.

100. Lamont RJ, Koo H, Hajishengallis G. The oral microbiota: dynamic communities and host interactions. Nature Reviews Microbiology. Nature Publishing Group; 2018;16:745-59. 


\section{RECIMA21 - REVISTA CIENTÍFICA MULTIDISCIPLINAR}

USO DE PROBIÓTICOS SISTÊMICOS NO TRATAMENTO PERIODONTAL NÃO CIRÚRGICO: REVISÃO NARRATIVA DA LITERATURA

Tabela 1. Comparativo entre os estudos clínicos publicados avaliando probióticos no tratamento periodontal

\begin{tabular}{cccc} 
ESTUDo CLíNICO & TIPO DE ESTUDO & $\begin{array}{c}\text { RESULTADOS } \\
\text { MICROBIOLÓGICOS }\end{array}$ & $\begin{array}{c}\text { RESULTADOS } \\
\text { IMUNOLÓGICOS }\end{array}$ \\
\hline Vivekanda 2010 & $\begin{array}{c}\text { Clínico } \\
\text { Aleatorizado }\end{array}$ & sim & não \\
Szkaradkiewicz et al., & Clínico Aleatorizado & sim & não \\
2014 & não & sim & \\
Ince et al., 2015 & Clínico Aleatorizado & não & sim \\
Laleman et al., 2015 & Clínico Aleatorizado & sim & não \\
Tecke et al., 2015 & Clínico Aleatorizado & sim & não \\
Morales et al., 2016 & Clínico Aleatorizado & não & não \\
Dhaliwal et al., 2017 & Clínico Aleatorizado & sim & não \\
Invernici et al., 2018 & Clínico Aleatorizado & sim & sim \\
Morales et al., 2018 & Clínico Aleatorizado & sim & não \\
Pelekos et al., 2019 & Clínico Aleatorizado & não & não \\
\hline
\end{tabular}




\section{RECIMA21 - REVISTA CIENTÍFICA MULTIDISCIPLINAR}

USO DE PROBIÓTICOS SISTÊMICOS NO TRATAMENTO PERIODONTAL NÃO CIRÚRGICO: REVISÃO NARRATIVA DA LITERATURA

Renata Queiroz Tavares, Mariana Nogueira de Figueiredo, Willy Bustillos Torrez, Cristiana Fernandes Plutarco Nogueira, Angelo Ferrari Roberto, Karyne Maria Rossit Silva Kiausinis, Magda Feres, Belén Retamal-Valdes

Tabela 2. Característica gerais dos estudos incluídos.

\begin{tabular}{|c|c|c|c|c|c|c|c|}
\hline REFERENCIA & $\mathbf{N}$ & PROBIOTICO & $\begin{array}{c}\text { FORMADE } \\
\text { APRESENTAÇÃO }\end{array}$ & $\begin{array}{c}\text { FORMADE } \\
\text { ADMINISTRAÇÃO }\end{array}$ & $\begin{array}{c}\text { TEMPO DE } \\
\text { ACOMPANHAMENTO }\end{array}$ & $\begin{array}{l}\text { RESULTADOS } \\
\text { CLÍNICOS }\end{array}$ & CONCLUSOES \\
\hline $\begin{array}{l}\text { Vivekananda et } \\
\text { al., 2010 }\end{array}$ & 30 & $\begin{array}{l}\text { Lactobacillus } \\
\text { reuteri }\end{array}$ & pastilha & $2 \mathrm{X}$ dia por 21 dias & 42 dias & $\begin{array}{l}\text { Redução estatística } \\
\text { de IP, IG, PS e NIC }\end{array}$ & $\begin{array}{l}\text { Sugere benefício } \\
\text { com uso de } \\
\text { probiótico }\end{array}$ \\
\hline $\begin{array}{l}\text { Teughels et al., } \\
2013\end{array}$ & 30 & $\begin{array}{l}\text { Lactobacillus } \\
\text { reuteri }\end{array}$ & pastilha & $\begin{array}{l}2 \times \text { dia por } 12 \\
\text { semanas }\end{array}$ & 12 semanas & $\begin{array}{l}\text { Redução estatística } \\
\text { de IP, IG, PS, NIC e } \\
\text { SS. }\end{array}$ & $\begin{array}{l}\text { Redução na PS e } \\
\text { NCl nas bolsas } \\
\text { moderadas e } \\
\text { profundas com o } \\
\text { uso rónticos. de } \\
\text { probiólios. }\end{array}$ \\
\hline $\begin{array}{l}\text { Szkaradkiewicz et } \\
\text { al., 2014 }\end{array}$ & 38 & $\begin{array}{l}\text { Lactobacillus } \\
\text { reuteri }\end{array}$ & pastilha & $\begin{array}{l}2 \times \text { dia por } 2 \\
\text { semanas }\end{array}$ & 30 dias & $\begin{array}{l}\text { Redução estatística } \\
\text { de SS, PS e NIC }\end{array}$ & $\begin{array}{l}\text { Sugere benefício } \\
\text { com uso de } \\
\text { probiótico }\end{array}$ \\
\hline Ince et al., 2015 & 30 & $\begin{array}{l}\text { Lactobacillus } \\
\text { reuteri }\end{array}$ & pastilha & $\begin{array}{l}2 \times \text { dia por } 3 \\
\text { semanas }\end{array}$ & 360 dias & $\begin{array}{l}\text { Redução estatística } \\
\text { de IP, IG, SS, PS }\end{array}$ & $\begin{array}{l}\text { Redução na PS } \\
\text { nas bolsas } \\
\text { moderadas com o } \\
\text { uso de probóticos. }\end{array}$ \\
\hline $\begin{array}{l}\text { Laleman et al., } \\
2015\end{array}$ & 48 & $\begin{array}{l}\text { Streptococcus } \\
\text { oralis } \mathrm{KJ} 3, \mathrm{~S} . \\
\text { uberis } \mathrm{KJ} 2, \mathrm{~S} . \\
\text { rattus } \mathrm{JH} 145\end{array}$ & pastilha & $\begin{array}{l}2 \times \text { dia por } 12 \\
\text { semanas }\end{array}$ & 24 semanas & $\begin{array}{l}\text { Redução estatística } \\
\text { apenas do IP. }\end{array}$ & $\begin{array}{l}\text { Não houve } \\
\text { benefício com o } \\
\text { uso de probiótico. }\end{array}$ \\
\hline Tekce et al., 2015 & 40 & $\begin{array}{l}\text { Lactobacillus } \\
\text { reuteri }\end{array}$ & pastilha & $\begin{array}{l}2 \times \text { dia por } 3 \\
\text { semanas }\end{array}$ & 360 dias & $\begin{array}{l}\text { Redução estatística } \\
\text { de IP, IG, SS e PS. }\end{array}$ & $\begin{array}{l}\text { Menor } \\
\text { necessidade de } \\
\text { cirurgia, menor } \\
\text { risco } \\
\text { progressão de da } \\
\text { doença com o uso } \\
\text { de probiótico. }\end{array}$ \\
\hline $\begin{array}{l}\text { Morales et al., } \\
2016\end{array}$ & 28 & $\begin{array}{l}\text { Lactobacillus } \\
\text { rhamnosus } \\
\text { SP1 }\end{array}$ & Sachet & $1 \mathrm{X}$ dia por 3 meses & 360 dias & $\begin{array}{l}\text { Redução na PS nas } \\
\text { bolsas moderadas e } \\
\text { profundas com o } \\
\text { uso de probióticos. }\end{array}$ & $\begin{array}{ll}\text { Menor risco } & \text { de } \\
\text { progressão } & \text { da } \\
\text { doença } & \\
\text { periodontal. } & \end{array}$ \\
\hline $\begin{array}{l}\text { Dhaliwal et al., } \\
2017\end{array}$ & 27 & $\begin{array}{l}\text { Streptococcus } \\
\text { faecalis, } \\
\text { Clostridium } \\
\text { butyricum, } \\
\text { Bacillus } \\
\text { mesentericus, } \\
\text { Lactobacillus } \\
\text { sporogenes I }\end{array}$ & pastilha & $2 \times$ dia por 21 dias & 90 dias & $\begin{array}{l}\text { Não houve } \\
\text { diferença estatística } \\
\text { nos parâmetros } \\
\text { avaliados }\end{array}$ & $\begin{array}{l}\mathrm{N} \text { ão houve } \\
\text { benefício com o } \\
\text { uso de probiótico. }\end{array}$ \\
\hline $\begin{array}{l}\text { Invernici et al., } \\
2018\end{array}$ & 41 & $\begin{array}{l}\text { Bifidobacterium } \\
\text { lactis (B. } \\
\text { lactis) HN019 }\end{array}$ & pastilha & $2 \times$ dia 30 dias & 90 dias & $\begin{array}{lr}\text { Menor } & \text { IP } \\
\text { dias. } & 30 \\
\text { moderadas } & \text { bolsas } \\
\text { profundas } & \text { maior } \\
\text { ganho NIC e menor } \\
\text { PS }\end{array}$ & $\begin{array}{l}\text { Menor } \\
\text { necessidade de } \\
\text { terapia adicional, } \\
\text { baixo risco de } \\
\text { progressão e } \\
\text { menor número de } \\
\text { bolsas residuais } \\
\text { com o uso de } \\
\text { probióticos. }\end{array}$ \\
\hline $\begin{array}{l}\text { Morales et al., } \\
2018\end{array}$ & 47 & $\begin{array}{l}\text { Lactobacillus } \\
\text { rhamnosus } \\
\text { SP1 }\end{array}$ & Sachet & $1 \mathrm{X}$ dia por 3 meses & 9 meses & $\begin{array}{l}\text { Não houve } \\
\text { diferença estatística } \\
\text { nos parâmetros } \\
\text { avaliados }\end{array}$ & $\begin{array}{l}\mathrm{N} \text { ão houve } \\
\text { benefício com o } \\
\text { uso de probiótico. }\end{array}$ \\
\hline $\begin{array}{l}\text { Pelekos et al., } \\
2019\end{array}$ & 41 & $\begin{array}{l}\text { Lactobacillus } \\
\text { reuteri }\end{array}$ & pastilha & $2 X$ dia 28 dias & 28 dias & $\begin{array}{lr}\text { Não houve } & \text { houve } \\
\text { diferença estatística } \\
\text { nos parâmetros } \\
\text { avaliados }\end{array}$ & $\begin{array}{l}\mathrm{N} \text { ão houve } \\
\text { benefício com o } \\
\text { uso de probiótico. }\end{array}$ \\
\hline
\end{tabular}




\section{RECIMA21 - REVISTA CIENTÍFICA MULTIDISCIPLINAR}

USO DE PROBIÓTICOS SISTÊMICOS NO TRATAMENTO PERIODONTAL NÃO CIRÚRGICO: REVISÃO NARRATIVA DA LITERATURA Renata Queiroz Tavares, Mariana Nogueira de Figueiredo, Willy Bustillos Torrez, Cristiana Fernandes Plutarco Nogueira, Angelo Ferrari Roberto, Karyne Maria Rossit Silva Kiausinis, Magda Feres, Belén Retamal-Valdes

Tabela 3. Resumo dos principais resultados clínicos obtidos nos estudos clínicos aleatorizados publicados testando probióticos sistêmicos adjuntos a terapia periodontal.

\begin{tabular}{|c|c|c|c|c|c|}
\hline Referência & $\begin{array}{l}\text { Profundidade de } \\
\text { sondagem (PS) }\end{array}$ & $\begin{array}{l}\text { Nível clínico de } \\
\text { inserção (NIC) }\end{array}$ & $\begin{array}{c}\text { índice gengival } \\
\text { (IG) }\end{array}$ & $\begin{array}{l}\text { Sangramento à } \\
\text { sondagem (SS) }\end{array}$ & $\begin{array}{l}\text { Acúmulo de } \\
\text { biofilme (AB) }\end{array}$ \\
\hline $\begin{array}{l}\text { Vivekananda et al., } \\
2010\end{array}$ & $\begin{array}{l}\text { Inicial: } 5.08+0.75 \\
\text { Final: } 3.78+0.61\end{array}$ & $\begin{array}{l}\text { Inicial: } 3.93+0.93 \\
\text { Final: } 2.85+0.74\end{array}$ & $\begin{array}{l}\text { Inicial: } 1.85+0.22 \\
\text { Final: } 1.01+0.10\end{array}$ & NÃO AVALIADO & $\begin{array}{c}\text { Inicial: } 1.79 \\
\text { Final: } 1.03+0.29\end{array}$ \\
\hline $\begin{array}{l}\text { Teughels et al., } \\
2013\end{array}$ & $\begin{array}{c}\text { Inícial: } 4.315+ \\
0.71 \\
\text { Final: } 2.73+0.71\end{array}$ & $\begin{array}{l}\text { Inicial: } 4.97+1.01 \\
\text { Final: } 3.97+0.97\end{array}$ & $\begin{array}{c}\text { Inicial: } 97.77+ \\
4.38 \\
\text { Final: } 4.3+10.69\end{array}$ & $\begin{array}{c}\text { Inicial: } 70.70+ \\
14.53 \\
\text { Final: } 15.51+ \\
11.92\end{array}$ & $\begin{array}{c}\text { Inicial: } 95+10.27 \\
\text { Final: } 16.34+ \\
19.19\end{array}$ \\
\hline $\begin{array}{c}\text { Szkaradkiewicz et } \\
\text { al., } 2014\end{array}$ & $\begin{array}{l}\text { Inicial: } 3.35+0.32 \\
\text { Final: } 3.06+0.27\end{array}$ & $\begin{array}{l}\text { Inicial: } 3.47+0.38 \\
\text { Final: } 3.16+0.27\end{array}$ & $\begin{array}{c}\text { Inicial: } 1.33+0.29 \\
\text { Final: } 1.21+0.36\end{array}$ & $\begin{array}{l}\text { Inicial: } 1.69+0.35 \\
\text { Final: } 1.24+0.31\end{array}$ & $\begin{array}{l}\text { Inicial: } 1.61+0.31 \\
\text { Final: } 1.65+0.26\end{array}$ \\
\hline Ince et al., 2015 & $\begin{array}{l}\text { Inicial: } 5.85+0.54 \\
\text { Final: } 1.70+0.31\end{array}$ & $\begin{array}{l}\text { Inicial: } 8.97+0.96 \\
\text { Final: } 1.39+0.26\end{array}$ & $\begin{array}{l}\text { Inicial: } 2.15+011 \\
\text { Final: } 11.60+4.35\end{array}$ & $\begin{array}{c}\text { Inicial: } 88.90+ \\
7.66 \\
\text { Final: } 0.73+0.28\end{array}$ & $\begin{array}{l}\text { Inicial: } 2.28+0.29 \\
\text { Final: } 0.76+0.24\end{array}$ \\
\hline $\begin{array}{l}\text { Laleman et al., } \\
\qquad 2015\end{array}$ & $\begin{array}{l}\text { Inicial: } 4.50+0.51 \\
\text { Final: } 2.90+0.47\end{array}$ & $\begin{array}{l}\text { Inicial: } 5.22+0.41 \\
\text { Final: } 5.53+0.27\end{array}$ & $\begin{array}{c}\text { Inicial: } 97.9 \\
\text { Final: } 26.98+9.34\end{array}$ & $\begin{array}{c}\text { Inicial: } 87.44+ \\
6.03 \\
\text { Final: } 44.0\end{array}$ & $\begin{array}{l}\text { Inicial: } 99.0 \\
\text { Final: } 51.7\end{array}$ \\
\hline Tekce et al., 2015 & $\begin{array}{l}\text { Inicial: } 5.23+0.68 \\
\text { Final: } 3.49+0.87\end{array}$ & NĀO AVALIADO & $\begin{array}{l}\text { Inicial: } 2.12+0.15 \\
\text { Final: } 11.05+3.99\end{array}$ & $\begin{array}{l}\text { Inicial: } 0.88+0.07 \\
\text { Final: } 0.80+0.38\end{array}$ & $\begin{array}{l}\text { Inicial: } 2.29+0.28 \\
\text { Final: } 0.73+0.24\end{array}$ \\
\hline $\begin{array}{l}\text { Morales et al., } \\
2016\end{array}$ & $\begin{array}{l}\text { Inicial: } 2.7+0.6 \\
\text { Final: } 2.1+0.5\end{array}$ & $\begin{array}{l}\text { Inicial: } 4.2+0.9 \\
\text { Final: } 4.1+1.0\end{array}$ & NÃO AVALIADO & $\begin{array}{l}\text { Inicial: } 41.1+16.3 \\
\text { Final: } 29.3+12.7\end{array}$ & $\begin{array}{l}\text { Inicial: } 63.1+18.5 \\
\text { Final: } 33.1+21.3\end{array}$ \\
\hline $\begin{array}{l}\text { Dhaliwal et al., } \\
\qquad 2017\end{array}$ & $\begin{array}{l}\text { Inicial: } 5.54 \pm 1.08 \\
\text { Final: } 4.62 \pm 1.32\end{array}$ & $\begin{array}{l}\text { Inicial: } 9.17 \pm 0.75 \\
\text { Final: } 8.16 \pm 1.55\end{array}$ & $\begin{array}{l}\text { Inicial: } 1.90 \pm 0.53 \\
\text { Final: } 1.49 \pm 0.35\end{array}$ & NÃO AVALIADO & $\begin{array}{c}\text { Inicial: } 1.59 \pm 0.34 \\
\text { Final: } 1.20 \pm 0.20\end{array}$ \\
\hline $\begin{array}{l}\text { Invernici et al., } \\
2018\end{array}$ & $\begin{array}{l}\text { Inicial: } 3.01 \\
\text { Final: } 2.49\end{array}$ & $\begin{array}{l}\text { Inicial: } 3.26 \\
\text { Final: } 2.77\end{array}$ & NĀO AVALIADO & $\begin{array}{l}\text { Inicial: } 30.80 \\
\text { Final: } 18.80\end{array}$ & $\begin{array}{l}\text { Inicial: } 23.85 \\
\text { Final: } 21.65\end{array}$ \\
\hline $\begin{array}{l}\text { Morales et al., } \\
2018\end{array}$ & $\begin{array}{l}\text { Inicial: } 2.7+0.6 \\
\text { Final: } 2.2+0.3\end{array}$ & $\begin{array}{l}\text { Inicial: } 3.8+0.7 \\
\text { Final: } 3.4+0.6\end{array}$ & NÃO AVALIADO & $\begin{array}{l}\text { Inicial: } 4.3+18.1 \\
\text { Final: } 42.4+14.6\end{array}$ & $\begin{array}{l}\text { Inicial: } 54.5+18.8 \\
\text { Final: } 28.1+14.6\end{array}$ \\
\hline $\begin{array}{l}\text { Pelekos et al., } \\
2019\end{array}$ & $\begin{array}{l}\text { Inicial: } 3.1 \\
\text { Final: } 2.6\end{array}$ & $\begin{array}{l}\text { Inicial: } 3.8 \\
\text { Final: } 3.7\end{array}$ & NĀO AVALIADO & $\begin{array}{l}\text { Inicial: } 63.6 \\
\text { Final: } 32.0\end{array}$ & $\begin{array}{l}\text { Inicial: } 37.0 \\
\text { Final: } 22.5\end{array}$ \\
\hline
\end{tabular}

